\title{
Elastic backscattering spectroscopic microscopy
}

\author{
Yang Liu, Xu Li, Young L. Kim, and Vadim Backman \\ Department of Biomedical Engineering, Northwestern University, Evanston, Illinois 60208
}

\begin{abstract}
Received April 22, 2005; revised manuscript received May 13, 2005; accepted May 24, 2005
The spectral properties of elastic light-scattering signals have been shown to provide a wealth of information on nanostructures and microstructures. We present elastic backscattering spectroscopic microscopy that allows simultaneous acquisition of microscopic images and backscattering spectra at each pixel. Within a single homogeneous micrometer-scale particle we observe two distinct and highly localized spectral oscillation features that arise from different optical paths: (1) surface waves (e.g., the ripple structure) and (2) a not previously reported anomalous ripple structure that is due to the interference of waves scattered from front and back surfaces at the particle's center. We also demonstrate that the spectroscopic data can provide nanoscale structural information beyond what conventional microscopy reveals. (C) 2005 Optical Society of America

OCIS codes: 170.0180, 170.6510, 290.1350, 170.3880.
\end{abstract}

Light-scattering signals from microscale-nanoscale structures exhibit characteristic structure-dependent patterns in wavelengths (i.e., spectrum) and scattering angles. It has been shown that these signatures, especially in the backward direction, are highly sensitive to subtle alterations in tissue architecture. ${ }^{1}$ In particular, recent studies have shown that elastic light-scattering spectroscopy may permit the earliest diagnosis of carcinogenesis known to date. ${ }^{2,3}$ In a typical elastic light-scattering spectroscopy experiment, a tissue area approximately several millimeters across is illuminated, and the information on the average size of scatterers within this area is obtained. However, in some applications it is desirable to obtain localized scattering signatures from either an isolated single scatterer or a localized structure within a complex multiparticle system. For example, in cytological analyses it is of clinical importance to detect a few cancer cells from a cell population. Furthermore, characterization of scattering from a localized structure may provide a convenient tool to explore the origin of scattering signatures from biological cells, which has not been fully understood partially due to the high complexity of cellular structures. Several investigators have developed techniques that incorporate the details of local microstructure with the scattering features, such as spectroscopic optical coherence tomography, ${ }^{3,4}$ spectral imaging, ${ }^{5}$ angle-dependent scattering, ${ }^{6,7}$ confocal spectroscopy, ${ }^{8}$ and microspectroscopy. ${ }^{9}$

In this Letter, we present elastic backscattering spectroscopic microscopy that takes advantage of both the diffraction-limited localized structural information provided by microscopy and the subdiffractional sensitivity of spectral signatures. We show that, for the first time to our knowledge, it is possible to identify the distinct and highly localized scattering features arising from different parts of a single homogeneous micrometer-scale particle, including surface scattering (i.e., scattering due to the wave creeping around the surface) and central scattering (i.e., scattering due to the wave traveling a round-trip optical path through the center of the particle).

Our elastic backscattering spectroscopic microscope simultaneously obtains a diffraction-limited resolution image of backscattered light and records the spectrum from each pixel. The design of the microscope is shown in Fig. 1(a). The broadband white light from a Xe lamp (100 W, Oriel) was collimated by a $4 f$ system (L1-A1-L2) with an $\sim 0.8^{\circ}$ full divergence angle and was focused onto a sample by a lownumerical-aperture (NA) objective (NA=0.4, Edmund Optics). The illumination beam diameter was $120 \mu \mathrm{m}$, which ensured that plane-wave illumination
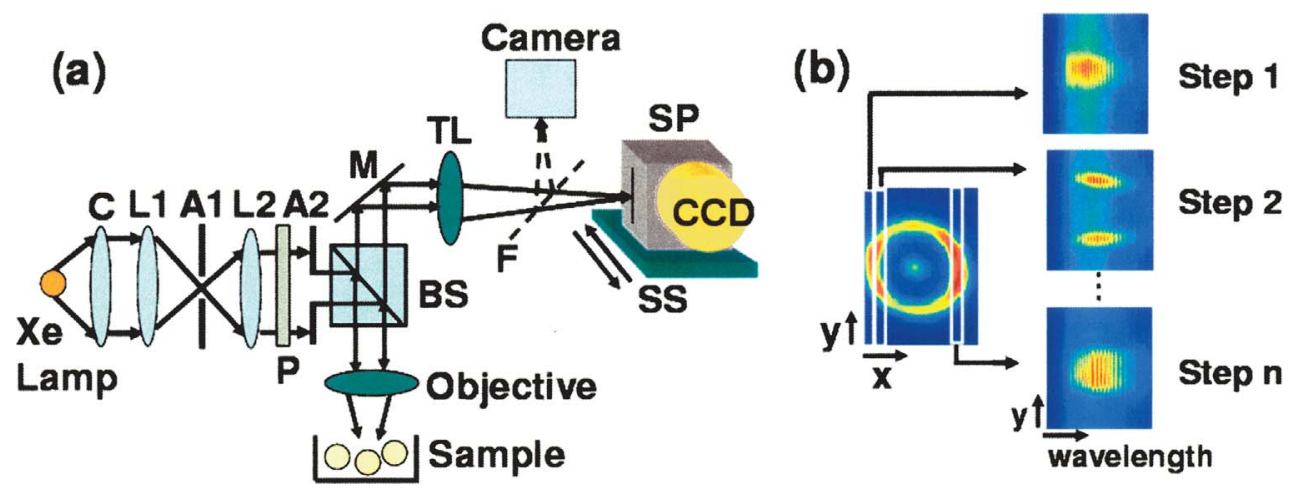

Fig. 1. Schematic of the elastic backscattering spectroscopic microscope system. (a) C, condenser; L1, L2, lenses $(f$ $=150 \mathrm{~mm})$; A1, A2, apertures $[D(\mathrm{~A} 1)=2 \mathrm{~mm}, D(\mathrm{~A} 2)=6 \mathrm{~mm}]$; BS, beam splitter; TL, tube lens $(f=450 \mathrm{~mm}) ; \mathrm{F}$, flipper; SS, scanning stage; SP, imaging spectrograph (slit width $10 \mu \mathrm{m}$, spectral resolution $\sim 3 \mathrm{~nm}$ ). (b) Simultaneous image and spectrum acquisition. 

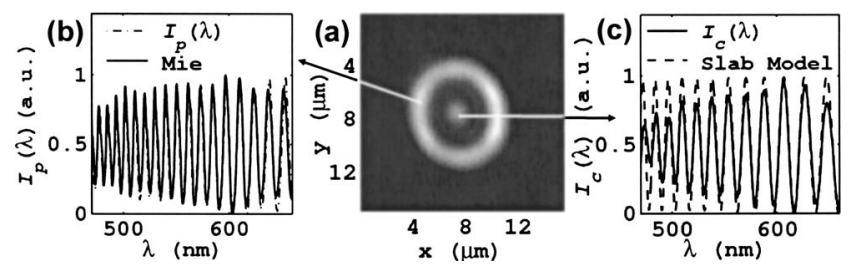

Fig. 2. (a) Microscopic image of a $6 \mu \mathrm{m}$ polystyrene microsphere embedded in agarose gel (gel concentration, $1 \%$; microsphere density, $\sim 8 \times 10^{5} \mathrm{particles} / \mathrm{ml}$ ). The focal plane is located approximately at the center of the sphere, and the sphere is $\sim 100 \mu \mathrm{m}$ below the surface. (b), (c) Representative scattering spectra recorded from (b) the periphery $I_{p}(\lambda)$ and (c) the center $I_{c}(\lambda)$ of the microsphere with Mie and slab model simulations, respectively.

could be assumed for analyzing light scattering signatures. The backscattered light was collected by the same objective and focused by a tube lens to form a magnified image. The imaging spectrograph (Acton Research) coupled with the CCD camera (Coolsnap $\mathrm{HQ}$, Roper Scientific) was mounted on a scanning stage (Zaber Technologies). The flipper mirror directed the image onto a digital camera for quick visualization of the image while adjusting the distance between the objective lens and the sample.

Figure 1(b) illustrates the data-acquisition procedure. The entrance slit of the imaging spectrograph was scanned along the $x$ axis of the image plane with a step size of $10 \mu \mathrm{m}$. In each scanning step, the CCD camera records a matrix with its $y$ axis corresponding to a spatial position along the $y$ axis of the image and $x$ axis corresponding to the wavelength of light. Thus the instrument records the final image with diffraction-limited spatial resolution by integrating the intensities of different wavelengths from the CCD. It is important that for each pixel of the final image there is a corresponding spectrum, which, as discussed below, provides a subdiffractional sensitivity $(\sim<50 \mathrm{~nm})$ for characterizing the structure of the scatterers beyond what is revealed by conventional microscopy.

To illustrate the capabilities of the instrument, we first used a single microsphere as a test model. Figure 2(a) shows a representative backscattering image of a $6 \mu \mathrm{m}$ polystyrene microsphere (Polysciences). The image shows two bright areas: in the periphery of the sphere and in its center. The same pattern was observed for microspheres with other sizes $(D=3.1$, $4.78,10.1 \mu \mathrm{m})$. The scattering spectra from the periphery $\left[I_{p}(\lambda)\right]$ and the center $\left[I_{c}(\lambda)\right]$ of the microsphere are shown in Figs. 2(b) and 2(c), respectively. Here $I(\lambda)$ is normalized by the spectral profile of the light source. The spectra exhibit the high-frequency oscillations known as the ripple structure characteristic of scattering by micrometer-sized particles. ${ }^{10}$ Since we focus on the high-frequency component of the spectrum, we removed the low-frequency component by using a high-pass filter to facilitate our analysis.

We first examine the spectral feature of periphery intensity $I_{p}(\lambda)$. The ripple structure was previously explained to arise from the interference effect be- tween the waves creeping around the surface ${ }^{10}$ (i.e., the surface wave) and can be predicted by far-field Mie theory, which is the analytical solution of light scattering by a single spherical particle. Indeed, there is a good match between $I_{p}(\lambda)$ and the Mie prediction, as shown in Fig. 2(b), indicating that far-field Mie scattering is dominated by surface scattering, in good agreement with previous theoretical explanation. We point out that these data provide experimental support for the origin of the backscattered ripple structure.

Interestingly, the ripple from the center oscillates much slower than one from the periphery [Fig. 2(c)], which cannot be predicted by the conventional farfield Mie theory. Furthermore, we note that this anomalous ripple structure $I_{c}(\lambda)$ is independent of the refractive indices of the surrounding media (data not shown), whereas Mie theory predicts that the ripple structure depends on the relative refractive index between the scatterer and the medium. We hypothesize that the origin of this a priori surprisingly slower oscillation pattern is the interference between the light reflected from the sphere-medium interface and that traveling a round-trip optical path through the center of the sphere. If our hypothesis is valid, the frequency and peak positions of spectrum should also be predicted from a uniform slab model $\left[I_{s}(\lambda)\right]$ with thickness equivalent to the sphere diameter. $I_{s}(\lambda)$ can be easily calculated analytically. ${ }^{11}$ As shown in Fig. 2(c), the spectral peaks in $I_{s}(\lambda)$ match well with those of $I_{c}(\lambda)$. The hypothesis was further confirmed by using a number of microspheres $(N=30)$ with different refractive indices $(n=1.59,1.46)$ and various diameters $D$ from 1.5 to $6 \mu \mathrm{m}$ (data not shown).

We also note that $I_{c}(\lambda)$ together with the simple slab model can be used to characterize the size of the particle with subdiffractional accuracy. We tested the sizing accuracy by using numerical data generated by the finite-difference time-domain (FDTD) method, since FDTD simulations can rigorously calculate scattering intensities at arbitrary spatial locations for well-defined geometries by solving the Maxwell's equations numerically. ${ }^{12}$ In our simulations, we incorporate the geometry of dielectric microspheres of various sizes into our FDTD grid by using a staircasing scheme with spatial resolution of $12.5 \mathrm{~nm}$. The scattered-field technique is employed to source a (a)

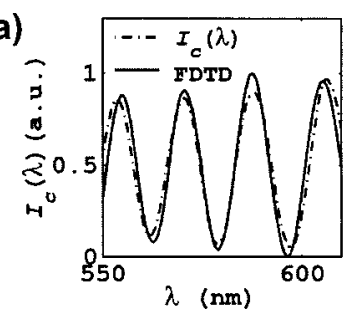

(b)

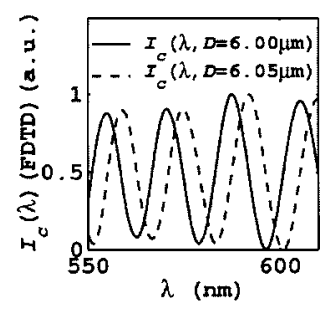

Fig. 3. (a) FDTD simulation, in agreement with $I_{c}(\lambda)$ from a microsphere experimentally measured by using the elastic backscattering spectroscopic microscope. (b) $I_{c}(\lambda)$ calculated by the FDTD method for two spheres with a $50 \mathrm{~nm}$ difference in diameter (6.00 and $6.05 \mu \mathrm{m}$, respectively). 

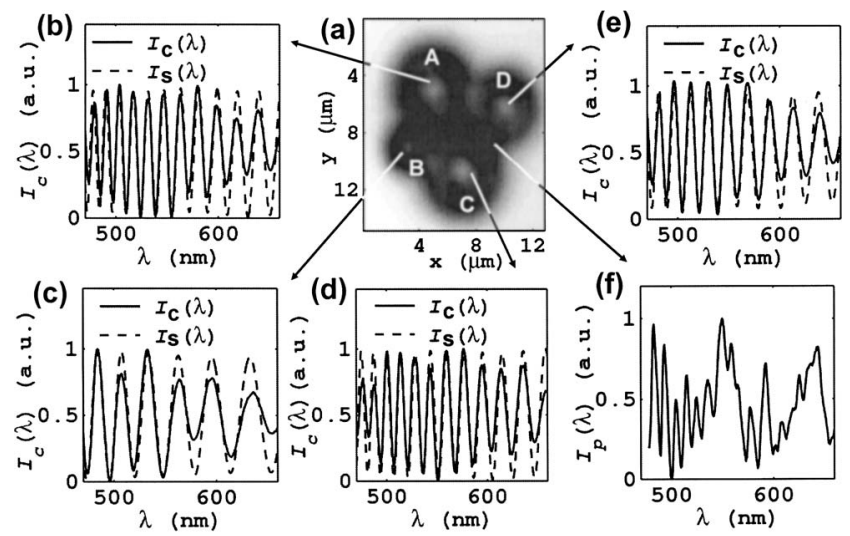

Fig. 4. (a) Representative microscopic image of aggregated polystyrene microspheres in air with mean diameters $D$ of A, $5.9 \mu \mathrm{m} ; \mathrm{B}, 3.1 \mu \mathrm{m}$; C, $5.9 \mu \mathrm{m} ; \mathrm{D}, 4.78 \mu \mathrm{m}$. (b)-(e) $I_{c}(\lambda)$ recorded from the four microspheres shown in panel (a) along with the slab model $I_{s}(\lambda)$, fitting (b) $I_{s}(\lambda, D$ $=5.935 \mu \mathrm{m})$, (c) $I_{s}(\lambda, D=3.095 \mu \mathrm{m})$, (d) $I_{s}(\lambda, D=5.90 \mu \mathrm{m})$, (e) $I_{s}(\lambda, D=4.73 \mu \mathrm{m})$. Evidently $I_{c}(\lambda)$ is not affected by multiple scattering, and it permits measurement of a particle size with an accuracy better than $\pm 50 \mathrm{~nm}$. On the other hand, as shown in (f), the conventional ripple structure $I_{p}(\lambda)$ (i.e., due to the surface wave) is affected by the interactions of neighboring particles.

time-domain pulsed plane wave that accommodates the complete frequency range of visible light. The center-scattering spectrum, $I_{c}(\lambda)$, is obtained by performing a discrete Fourier transform of the timedomain field intensity at an observation point located on the optical path through the center of the microsphere and normalized by the source spectrum. First we validate our numerical simulation by comparing them with experimental results for a microsphere with $D=6 \mu \mathrm{m}$. As shown in Fig. 3(a), our FDTD simulation accurately predicts $I_{c}(\lambda)$ obtained experimentally. Then we slightly vary the size of the microsphere in our FDTD model to test the sensitivity of $I_{c}(\lambda)$ 's dependence on particle size. Figure 3(b) shows that FDTD-calculated $I_{c}(\lambda)$ can clearly distinguish two microspheres with an only $50 \mathrm{~nm}$ difference in diameter. Furthermore, we note that the peak position of $I_{c}(\lambda)$ for a sphere can be fitted well by the slab model with the same refractive index and a thickness equivalent to the sphere diameter. These results demonstrate that our technique is capable of predicting particle size with subdiffractional nanoscale accuracy.

We point out that this technique not only allows assessment of an isolated scatterer but also permits identification of the localized scattering signatures within a complex system. We used aggregated polystyrene microspheres with different sizes as our model, as shown in Fig. 4(a). Evidently the microscopic image cannot provide an accurate measure of their individual sizes. On the other hand, as shown in Figs. 4(b)-4(e), despite the fact that the particles are densely packed, their individual single scattering features $I_{c}(\lambda)$ can be clearly discerned without being affected by the multiple scattering between particles. The particle sizes can be accurately obtained with a standard error $<50 \mathrm{~nm}$ by fitting the spectra predicted by the slab model $I_{s}(\lambda)$ to $I_{c}(\lambda)$ by using a leastsquares minimization algorithm. In addition, $I_{c}(\lambda)$ is independent of the refractive index of the surrounding medium (data not shown), which significantly facilitates the detection of scattering signatures from a single particle. In comparison, the surface scattering [Fig. 4(f)] is affected by the refractive index of the medium and multiple scattering interactions between neighboring particles.

In conclusion, we have developed elastic backscattering spectroscopic microscopy, which enables detection of highly localized scattering features within a single scattering particle as well as densely packed scatterers not obstructed by multiple scattering. In the future this technique may be used for characterization of internal structures of living or fixed cells as part of a cytological preparation, for identification of spectroscopic markers associated with disease-specific cellular changes, and to help us understand the origin of light scattering signals and image formation in several modalities of optical microscopy and spectroscopic optical coherence tomography.

This study was supported by National Science Foundation (NSF) grants BES0238903 and BES0417689. The computer resource is supported by NSF Teragrid under project TG-MCB 040062N. We acknowledge P. Pradhan, J. Gong, and A. Taflove for helpful discussions. Y. Liu's email address is yangliu@northwestern.edu.

\section{References}

1. X. Li, Z. G. Chen, A. Taflove, and V. Backman, Proc. SPIE 5693, 92 (2005).

2. H. K. Roy, Y. Liu, R. K. Wali, Y. L. Kim, A. K. Kromine, M. J. Goldberg, and V. Backman, Gastroenterology 126, 1071 (2004).

3. X. D. Li, S. A. Boppart, J. Van Dam, H. Mashimo, M. Mutinga, W. Drexler, M. Klein, C. Pitris, M. L. Krinsky, M. E. Brezinski, and J. G. Fujimoto, Endoscopy 32, 921 (2000).

4. A. Wax, C. H. Yang, and J. A. Izatt, Opt. Lett. 28, 1230 (2003)

5. E. Schrock, S. du Manoir, T. Veldman, B. Schoell, J. Wienberg, M. A. Ferguson-Smith, Y. Ning, D. H. Ledbetter, I. Bar-Am, D. Soenksen, Y. Garini, and T. Ried, Science 273, 494 (1996).

6. M. T. Valentine, A. K. Popp, D. A. Weitz, and P. D. Kaplan, Opt. Lett. 26, 890 (2001).

7. N. N. Boustany, S. C. Kuo, and N. V. Thakor, Opt. Lett. 26, 1063 (2001).

8. G. Schuele, E. Vitkin, P. Huie, C. O’Conell-Rodwell, D. Palanker, and D. L. T. Perelman "Optical spectroscopy non-invasively monitors response of organelles to cellular stress," J. Biomed. Opt. (to be published).

9. A. Curry, G. Nusz, A. Chilkoti, and A. Wax, Opt. Express 13, 2668 (2005).

10. H. C. van de Hulst, Light Scattering by Small Particles (Dover, 1981).

11. P. W. Barber and S. C. Hill, Light Scattering by Particles: Computational Methods, Advanced Series in Applied Physics (World Scientific, 1990).

12. A. Taflove and S. C. Hagness, Computational Electrodynamics: the Finite-Difference Time-Domain Method (Artech, 2000). 